\title{
Article \\ Occurrence of Multi-Drug-Resistant Escherichia coli in Chickens, Humans, Rodents and Household Soil in Karatu, Northern Tanzania
}

\author{
Valery S. Sonola ${ }^{1,2,3, *}$, Abdul S. Katakweba ${ }^{1,4}$, Gerald Misinzo ${ }^{5}\left({ }^{-}\right.$and Mecky I. N. Matee ${ }^{6}$ \\ 1 Africa Centre of Excellence for Innovative Rodent Pest Management and Biosensor Technology \\ Development (ACE IRPM \& BTD), Sokoine University of Agriculture, P.O. Box 3110, \\ Morogoro 67125, Tanzania; katakweba@sua.ac.tz \\ 2 Department of Wildlife Management, College of Forestry, Wildlife and Tourism, P.O. Box 3073, \\ Morogoro 67125, Tanzania \\ 3 Livestock Training Agency (LITA), Buhuri Campus, P.O. Box 1483, Tanga 21206, Tanzania \\ 4 Institute of Pest Management, Sokoine University of Agriculture, P.O. Box 3110, Morogoro 67125, Tanzania \\ 5 Department of Veterinary Microbiology, Parasitology and Biotechnology, College of Veterinary Medicine and \\ Biomedical Sciences, Sokoine University of Agriculture, P.O. Box 3019, Morogoro 67125, Tanzania; \\ gerald.misinzo@sacids.org \\ 6 Department of Microbiology and Immunology, School of Medicine, Muhimbili University of Health and \\ Allied Sciences, P.O. Box 65001, Dar es Salaam 11103, Tanzania; mateemecky@yahoo.com \\ * Correspondence: vssonola@gmail.com
}

Citation: Sonola, V.S.; Katakweba, A.S.; Misinzo, G.; Matee, M.I.N.

Occurrence of Multi-Drug-Resistant Escherichia coli in Chickens, Humans, Rodents and Household Soil in Karatu, Northern Tanzania. Antibiotics 2021, 10, 1137. https:// doi.org/10.3390/antibiotics10091137

Academic Editor: William R. Schwan

Received: 9 July 2021

Accepted: 13 September 2021

Published: 21 September 2021

Publisher's Note: MDPI stays neutral with regard to jurisdictional claims in published maps and institutional affiliations.

Copyright: (C) 2021 by the authors. Licensee MDPI, Basel, Switzerland. This article is an open access article distributed under the terms and conditions of the Creative Commons Attribution (CC BY) license (https:// creativecommons.org/licenses/by/ $4.0 /)$.

\begin{abstract}
We investigated antibiotic resistance profiles of Escherichia coli among 960 samples obtained from chickens (236), humans (243), rodents (101) and soil (290). E. coli was isolated from 650 (67.7\%) samples. Isolation frequency varied significantly between chickens, humans, rodents and soil samples, being $81.6 \%, 86.5 \%, 79.2 \%$ and $31.0 \%$, respectively $(p<0.001)$. Resistance rates were particularly higher against imipenem (79.8\%), cefotaxime (79.7\%) and tetracycline $(73.7 \%)$ and moderate against amoxicillin-clavulanate (49.4\%). Overall, $78.8 \%$ of the isolates were multidrug-resistant (MDR) among which, $38.8 \%, 25.1 \%, 12.9 \%$ and $2.5 \%$ exhibited resistance to three, four, five and six different classes of antibiotics, respectively. Multidrug-resistant E. coli were observed in $27.7 \%, 30.3 \%, 10.8 \%$ and $10.0 \%$ of the isolates from chickens, humans, rodents and soil samples, respectively. Our results show high levels of antimicrobial resistance including MDR in E. coli isolated from chickens, humans, rodents and soil samples in Karatu, Northern Tanzania. Comprehensive interventions using a one-health approach are needed and should include improving (i) awareness of the community on judicious use of antimicrobial agents in humans and animals, (ii) house conditions and waste management and (iii) rodent control measures.
\end{abstract}

Keywords: Escherichia coli; antibiotic resistance; humans; rodents; chickens; soil; isolates

\section{Introduction}

Antibiotic resistance is currently a serious problem worldwide that threatens human, animal and environmental health [1]. If the situation remains unmanaged by 2050, higher human mortalities, severe economic losses and a significant drop in livestock production are expected [2]. Escherichia coli is the major cause of urinary tract infections and neonatal meningitis in humans [3], and it also causes avian colibacillosis, a serious infectious disease in poultry [4]. Other conditions caused by E. coli in chickens include yolk sac infections, pericarditis, peritonitis and osteomyelitis [5]. E. coli is a commensal microbe in humans and chickens that carries and spreads resistance genes to other pathogens [6], threatening public health. Rodents that invade human habitats carry and transmit different zoonotic pathogens including MDR E. coli, threatening human health [7,8]. The interaction between rodents, humans, livestock and their environment in households can facilitate sharing of antibiotic-resistant bacteria and their resistance genes [9]. Studies have documented that, 
in rural areas where poultry farming is common, household soils are contaminated with higher antibiotic residues from humans and animals [10-13], leading to an increase in and spread of antibiotic resistance genes, involving contamination of the environment [14]. Previous studies have pointed out the potential role of peridomestic rodents in the spread of resistant bacteria to humans and domestic animals, either directly or through the environment $[7,14,15]$. Thus, the interaction between humans, livestock and peridomestic rodents is of public health concern, since it has the potential to cause infections that are difficult to treat [9]. In Karatu district in Northern Tanzania, studies have shown intense interactions between rodents, humans and animals, leading to infectious disease epidemics [16-20]. However, none of these studies screened bacteria for antimicrobial resistance. We hypothesize these interactions can cause the transmission of resistant bacteria, fueling the spread of antimicrobial resistance (AMR) in the community. We conducted this study in Karatu district in the northern zone of Tanzania to isolate and phenotypically screen for antimicrobial resistant E. coli among humans, chickens, rodents and the soil in households that keep indigenous chickens. This cross-sectional study determined phenotypic AMR patterns of E. coli isolated from rodents, humans, chickens, and their environment in households in an area where their interaction is intense.

\section{Results}

\subsection{Isolation of Escherichia coli from the Samples}

E. coli was isolated from $650(67.7 \%)$ samples (Table 1). Isolation of E. coli varied significantly between chickens, humans, rodents and soil samples at $81.9 \%, 86.5 \%, 80.2 \%$ and $31.0 \%$, respectively $(p<0.001)$.

Table 1. Isolation frequencies of Escherichia coli from different samples.

\begin{tabular}{ccccc}
\hline $\begin{array}{c}\text { Type of } \\
\text { Samples }\end{array}$ & $\begin{array}{c}\text { Total Number of } \\
\text { Samples n (\%) }\end{array}$ & $\begin{array}{c}\text { Positive } \\
\text { Samples n (\%) }\end{array}$ & Chi-Square & $p$-Value \\
\hline Chickens & $288(30.0)$ & $236(81.9)$ & & \\
Humans & $281(29.3)$ & $243(86.5)$ & & \\
Rodents & $101(10.5)$ & $81(80.2)$ & $X^{2}=147.58, \mathrm{df}=3$ & $<0.001$ \\
Soil & $290(30.2)$ & $90(31.0)$ & & \\
Total & $960(100.0)$ & $650(67.7)$ & & \\
\hline
\end{tabular}

\subsection{Antibiotic Resistance of E. coli Isolates from Chickens, Humans, Rodents and Soil}

Overall, the E. coli isolates were resistant to tetracycline $(73.7 \%)$, amoxicillin-clavulanate (49.4\%), imipenem (79.8\%), ciprofloxacin (40.2\%), cefotaxime $(79.7 \%)$ and gentamycin $(9.7 \%)$, as shown in Figure 1. The overall resistance rates were 34.5\%, 38.6\%, 13.7\% and $13.1 \%$ for isolates from chickens, humans, rodents and soil, respectively. Increased resistance of isolates to imipenem, cefotaxime and tetracycline was observed in all types of samples (Figure 1).

\subsection{Multidrug-Resistance (MDR) of Escherichia coli Isolates from All Samples}

A total of 512 out of 650 isolates $(78.8 \%)$, were resistant to three and above different classes of antibiotics. Most of the isolates (38.3\%) were resistant to three classes of antibiotics, and 16 isolates $(2.5 \%)$ were resistant to all classes where most of them were from chicken (seven isolates) and human (five isolates) samples (Table 2). We observed significant variation in the occurrence of MDR isolates from different types of samples being higher in humans (30.3\%) and chickens (27.7\%) compared to rodents $(10.8 \%)$ and soils $(10.0 \%)(p<0.001)$. 


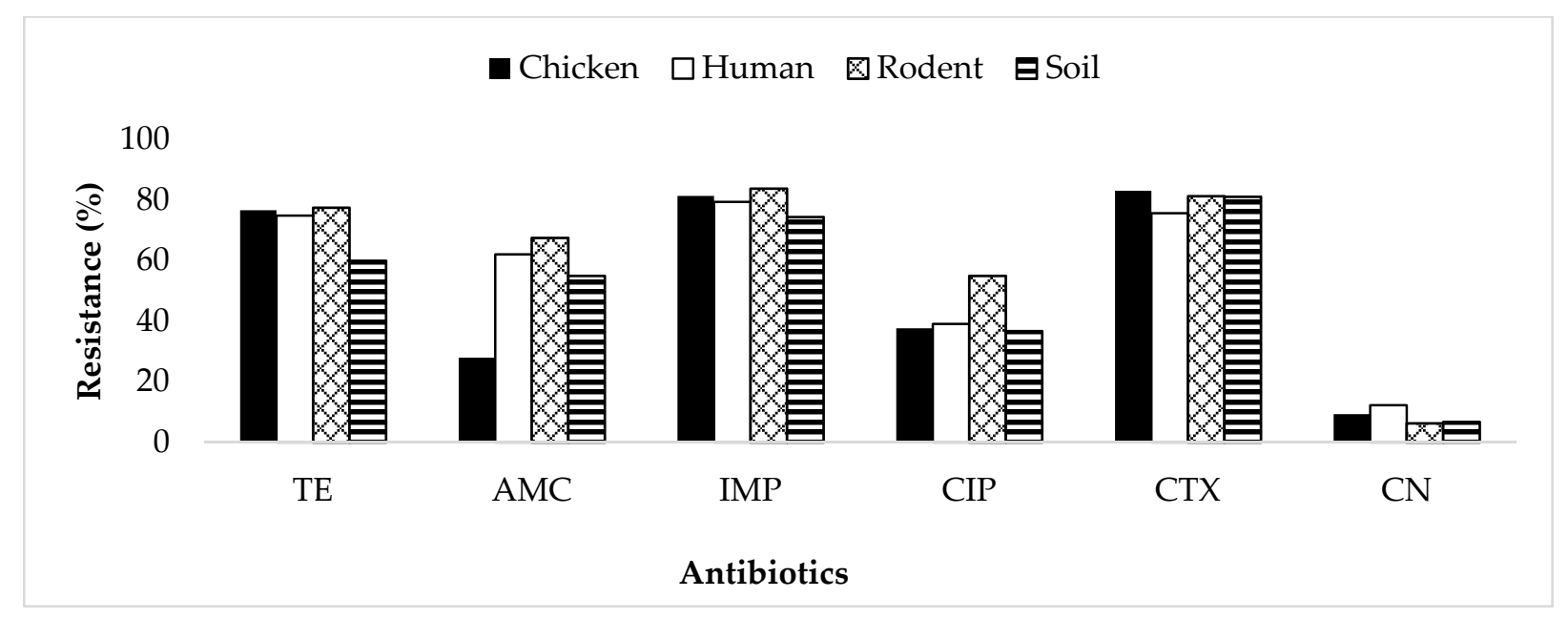

Figure 1. Resistance rates of $E$. coli isolates to different classes of antibiotics. TE = tetracycline; AMC = amoxicillinclavulanate; IMP = imipenem; $\mathrm{CIP}=$ ciprofloxacin; $\mathrm{CTX}=$ cefotaxime; $\mathrm{CN}$ = gentamycin.

Table 2. Antibiotic resistance and MDR rates of Escherichia coli isolates from chickens, humans, rodents and soil samples.

\begin{tabular}{|c|c|c|c|c|c|c|c|c|c|c|c|}
\hline \multirow{2}{*}{$\begin{array}{l}\text { Types of } \\
\text { Sample } \\
\text { Sources }\end{array}$} & \multicolumn{7}{|c|}{$\begin{array}{l}\text { Number of Antibiotic Classes to Which the Isolates Were Resistant, } \\
\text { n (\%) }\end{array}$} & \multirow{2}{*}{$\begin{array}{c}\text { Total } \\
\text { Number } \\
\text { of Isolates }\end{array}$} & \multirow{2}{*}{$\begin{array}{c}\text { MDR } \\
\text { Isolates } \\
\text { (3-6 Classes) }\end{array}$} & \multirow{2}{*}{$\begin{array}{l}\text { Chi- } \\
\text { Square }\end{array}$} & \multirow{2}{*}{$\begin{array}{c}p- \\
\text { Value }\end{array}$} \\
\hline & 0 & 1 & 2 & 3 & 4 & 5 & 6 & & & & \\
\hline $\begin{array}{l}\text { Overall } \\
\mathrm{n}(\%)\end{array}$ & $5(0.8)$ & $24(3.7)$ & $\begin{array}{c}109 \\
(16.8)\end{array}$ & $\begin{array}{c}249 \\
(38.3)\end{array}$ & $\begin{array}{c}163 \\
(25.1)\end{array}$ & 84 (12.9) & $16(2.5)$ & $650(100.0)$ & $512(78.8)$ & & \\
\hline Chickens & $0(0.0)$ & $10(1.5)$ & $46(7.1)$ & $\begin{array}{c}103 \\
(15.8)\end{array}$ & $55(8.5)$ & $15(2.3)$ & $7(1.1)$ & $236(36.3)$ & $180(27.7)$ & $\begin{array}{l}129.75 \\
\mathrm{df}=3\end{array}$ & $<0.001$ \\
\hline Humans & $5(0.8)$ & $8(1.2)$ & $33(5.1)$ & 88 (13.5) & $63(9.7)$ & $41(6.3)$ & $5(0.8)$ & $243(37.4)$ & $197(30.3)$ & $\begin{array}{l}75.47 \\
\mathrm{df}=3\end{array}$ & $<0.001$ \\
\hline Rodents & $0(0.0)$ & $2(0.3)$ & $9(1.4)$ & $25(3.8)$ & $20(3.1)$ & $22(3.4)$ & $3(0.5)$ & 81 (12.5) & 70 (10.8) & $\begin{array}{l}16.16 \\
\mathrm{df}=3\end{array}$ & 0.001 \\
\hline Soil & $0(0.0)$ & $4(0.6)$ & $21(3.2)$ & $33(5.1)$ & $25(3.8)$ & $6(0.9)$ & $1(0.2)$ & $90(13.8)$ & $65(10.0)$ & $\begin{array}{l}42.75 \\
\mathrm{df}=3\end{array}$ & $<0.001$ \\
\hline
\end{tabular}

\subsection{Prevalence of MDR Isolates of E. coli in Different Locations of Karatu}

Among 512 MDR isolates, 74, 81, 153, 137 and 116 were isolated from the samples collected in Endabash, Endamarariek, Karatu, Mbulumbulu and Rhotia wards, respectively (Figure 2).

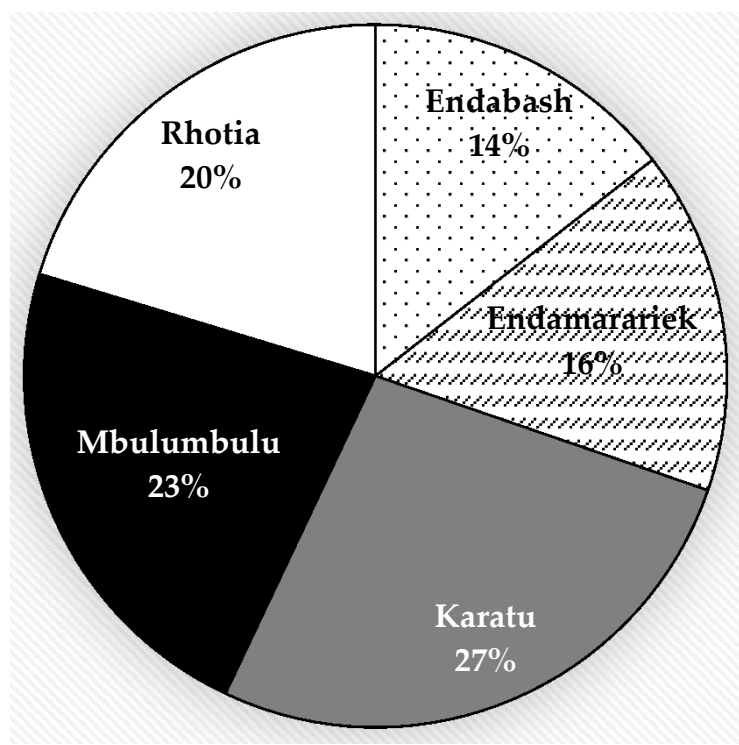

Figure 2. Distribution of MDR E. coli isolates in different areas of Karatu district. 
All MDR E. coli isolates from chickens, humans, rodents and soil samples were distributed in the wards as shown in Table 3. Significant variation in the prevalence of MDR isolates was observed between the wards, being higher in samples from Karatu (21.1\%) compared to those from Endabash $(11.4 \%)$ ward $(p<0.001)$ (Table 3).

Table 3. Prevalence of MDR E. coli isolates from all types of samples in different wards in Karatu district.

\begin{tabular}{|c|c|c|c|c|c|c|c|}
\hline \multirow{2}{*}{ Ward } & \multicolumn{4}{|c|}{ Types of Sample Sources, n (\%) } & \multirow{2}{*}{$\begin{array}{c}\text { MDR } \\
\text { Isolates }\end{array}$} & \multirow{2}{*}{ Chi-Square } & \multirow{2}{*}{$p$-Value } \\
\hline & Chickens & Humans & Rodents & Soil & & & \\
\hline Overall n (\%) & $180(27.7)$ & $197(30.3)$ & $70(10.8)$ & $65(10.0)$ & $512(78.8)$ & & \\
\hline Endabash & $20(3.1)$ & $22(3.4)$ & $17(2.6)$ & $15(2.3)$ & $74(11.4)$ & $1.6571, \mathrm{df}=3$ & 0.647 \\
\hline Endamarariek & $32(4.9)$ & $27(4.2)$ & $10(1.5)$ & $12(1.8)$ & $81(12.5)$ & $18.532, \mathrm{df}=3$ & $<0.001$ \\
\hline Karatu & $40(6.2)$ & $61(9.4)$ & $24(3.7)$ & $12(1.8)$ & $137(21.1)$ & $49.118, \mathrm{df}=3$ & $<0.001$ \\
\hline Mbulumbulu & $50(7.7)$ & $42(6.5)$ & $8(1.8)$ & $16(2.5)$ & $116(17.8)$ & $43.571, \mathrm{df}=3$ & $<0.001$ \\
\hline Rhotia & $38(5.8)$ & $45(6.9)$ & $11(1.7)$ & $10(1.5)$ & $104(16.0)$ & $39.44, \mathrm{df}=3$ & $<0.001$ \\
\hline $\begin{array}{c}\text { Chi-Square } \\
p \text {-value }\end{array}$ & $\begin{array}{c}24.82, \mathrm{df}=4 \\
<0.001\end{array}$ & $\begin{array}{c}25.03, \mathrm{df}=4 \\
<0.001\end{array}$ & $\begin{array}{c}13.077, \mathrm{df}=4 \\
0.011\end{array}$ & $\begin{array}{c}2.00, \mathrm{df}=4 \\
0.736\end{array}$ & & & \\
\hline
\end{tabular}

As shown in Figure 3, along principal component 1 (PC1), the arrow for gentamycin $(\mathrm{CN})$ is very close to 0 (X-axis), followed by that of ciprofloxacin (CIP), indicating their respective lower variances compared to other drugs, indicating that isolates were more susceptible to $\mathrm{CN}$ followed by CIP. The arrow for amoxicillin-clavulanate (AMC) is far (high deviation) from the $\mathrm{PC} 1$ axis (Susceptibility), indicating relatively higher resistance rates of isolates to AMC compared to $\mathrm{CN}$ and $\mathrm{CIP}$. Along the principal component 2 (PC2), the arrows for tetracycline (TE), imipenem (IMP), cefotaxime (CTX) and AMC are close to PC2 ( $Y$-axis), showing higher variances compared to other drugs, which implies higher resistance rates of isolates to these drugs. The large positive loadings for TE, IMP and CTX indicate a greater and positive correlation between them in terms of resistance patterns. The overlapping of ellipses indicates that the proportions of resistant isolates did not vary significantly across sample sources.

\subsection{Phenotypic Patterns of MDR E. coli Isolates from Chickens, Humans, Rodents and Soil}

MDR E. coli isolates from chickens displayed different resistance patterns where TEIMP-CTX (7.4\%), TE-IMP-CIP-CTX (3.5\%) and TE-AMC-CIP-CTX (3.1\%) were the most common as shown in Table 4.

MDR isolates from human samples displayed TE-IMP-CTX (4.5\%), TE-AMC-IMP-CTX (4.9\%) and TE-AMC-IMP-CIP-CTX (5.1\%) as the common patterns of resistance (Table 5).

Among 70 MDR E. coli isolates from rodent samples, 8 isolates (4.5\%), 9 isolates $(1.4 \%)$ and 22 isolates (3.4\%) displayed TE-IMP-CTX, TE-AMC-IMP-CTX and TE-AMC-IMP-CIPCTX as common patterns of resistance, respectively (Table 6).

For MDR isolates from soil samples, TE-IMP-CTX (2.0\%) and TE-AMC-IMP-CTX $(1.8 \%)$ were the common resistance patterns, as shown in Table 7 below. Overall, the combination TE-IMP-CTX was the most common pattern appearing in isolates from all types of samples. 


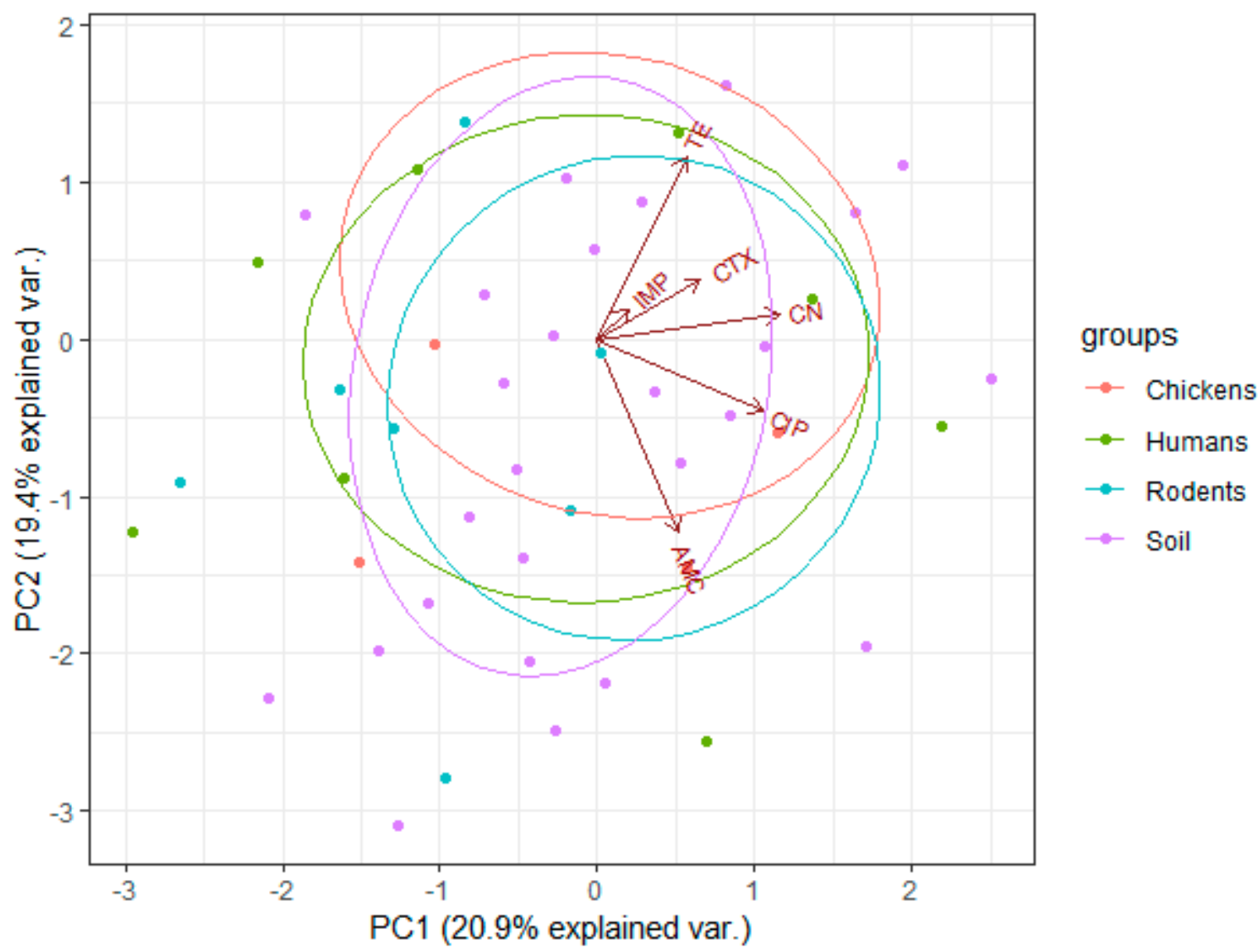

Figure 3. Principal component analysis (PCA) biplots of the antibiotic resistance profiles of $E$. coli isolated from chickens, humans, rodents and soil samples. For principal component 1 (PC1), the $X$-axis expresses susceptibility of the isolates to the drugs, while for principal component 2 (PC2), the $Y$-axis expresses resistance of the isolates. Arrows indicate the antibiotics that were used during resistance screening. The dots represent the E. coli isolates that were resistant to the tested antibiotics with respect to their sample sources. The ellipses indicate a $95 \%$ confidence interval of the respective isolates from the same sample source.

Table 4. Resistance patterns of 180 MDR E. coli isolates from chicken samples.

\begin{tabular}{|c|c|c|c|c|}
\hline Chicken Samples & Number of Isolates (n) & Occurrence (\%) & $\begin{array}{c}\text { Antibiotic Resistance } \\
\text { Patterns }\end{array}$ & $\begin{array}{c}\text { Number of } \\
\text { Antibiotic Classes }\end{array}$ \\
\hline$(n=180)$ & $\begin{array}{c}10 \\
9 \\
1 \\
2 \\
3 \\
2 \\
4 \\
48 \\
1 \\
2 \\
12 \\
2 \\
3 \\
2 \\
1 \\
1\end{array}$ & $\begin{array}{l}1.5 \\
1.4 \\
0.2 \\
0.3 \\
0.5 \\
0.3 \\
0.6 \\
7.4 \\
0.2 \\
0.3 \\
1.8 \\
0.3 \\
0.5 \\
0.3 \\
0.2 \\
0.2\end{array}$ & $\begin{array}{c}\text { AMC, IMP, CTX } \\
\text { IMP, CIP, CTX } \\
\text { AMC, IMP, CIP } \\
\text { AMC, CIP, CTX } \\
\text { TE, AMC, CTX } \\
\text { TE, AMC, IMP } \\
\text { TE, CIP, CTX } \\
\text { TE, IMP, CTX } \\
\text { TE, IMP, CIP } \\
\text { TE, AMC, CTX } \\
\text { TE, CIP, CTX } \\
\text { TE, IMP, CIP } \\
\text { TE, CIP, CTX } \\
\text { TE, AMC, IMP } \\
\text { TE, IMP, CN } \\
\text { TE, AMC, CIP }\end{array}$ & 3 \\
\hline
\end{tabular}


Table 4. Cont.

\begin{tabular}{|c|c|c|c|c|}
\hline Chicken Samples & Number of Isolates (n) & Occurrence (\%) & $\begin{array}{c}\text { Antibiotic Resistance } \\
\text { Patterns }\end{array}$ & $\begin{array}{c}\text { Number of } \\
\text { Antibiotic Classes }\end{array}$ \\
\hline & 1 & 0.2 & TE, CIP, CTX, CN & \multirow{8}{*}{4} \\
\hline & 2 & 0.3 & AMC, IMP, CTX, CN & \\
\hline & 4 & 0.6 & AMC, IMP, CIP, CTX, & \\
\hline & 23 & 3.5 & TE, IMP, CIP, CTX & \\
\hline & 1 & 0.2 & TE, IMP, CIP, CN & \\
\hline & 20 & 3.1 & TE, AMC, CIP, CTX & \\
\hline & 2 & 0.3 & TE, AMC, IMP, CTX & \\
\hline & 2 & 0.3 & TE, IMP, CTX, CN & \\
\hline & 10 & 1.5 & TE, AMC, IMP, CIP, CTX & \multirow{4}{*}{5} \\
\hline & 3 & 0.5 & TE, IMP, CIP, CTX, CN & \\
\hline & 1 & 0.2 & TE, AMC, IMP, CTX, CN & \\
\hline & 1 & 0.2 & TE, IMP, CIP, CTX, CN & \\
\hline & 7 & 1.1 & TE, AMC, IMP, CIP, CTX, CN & 6 \\
\hline Total & 180 & 27.7 & & \\
\hline
\end{tabular}

$\mathrm{AMC}=$ amoxicillin-clavulanate; $\mathrm{TE}=$ tetracycline; $\mathrm{IMP}=$ imipenem $; \mathrm{CTX}=$ cefotaxime; $\mathrm{CIP}=$ ciprofloxacin $; \mathrm{CN}=$ gentamycin .

Table 5. Resistance patterns of 197 MDR E. coli isolates from human samples.

\begin{tabular}{|c|c|c|c|c|}
\hline Human Samples & Number of Isolates (n) & Occurrence $(\%)$ & $\begin{array}{l}\text { Antibiotic Resistance } \\
\text { Patterns }\end{array}$ & $\begin{array}{c}\text { Number of } \\
\text { Antibiotic Classes }\end{array}$ \\
\hline \multirow[t]{30}{*}{$(\mathrm{n}=197)$} & 17 & 2.6 & AMC, IMP, CTX & \multirow{14}{*}{3} \\
\hline & 5 & 0.8 & AMC, IMP, CIP & \\
\hline & 2 & 0.3 & AMC, CIP, CTX & \\
\hline & 29 & 4.5 & TE, IMP, CTX & \\
\hline & 10 & 1.5 & TE, AMC, CTX & \\
\hline & 1 & 0.2 & IMP, CIP, CTX & \\
\hline & 8 & 1.2 & TE, AMC, IMP & \\
\hline & 7 & 1.1 & TE, IMP, CIP & \\
\hline & 2 & 0.3 & TE, AMC, CIP & \\
\hline & 2 & 0.3 & TE, CIP, CTX & \\
\hline & 2 & 0.3 & $\mathrm{TE}, \mathrm{CTX}, \mathrm{CN}$ & \\
\hline & 1 & 0.2 & TE, IMP, CTX & \\
\hline & 1 & 0.2 & TE, CIP, CTX & \\
\hline & 1 & 0.2 & TE, IMP, CN & \\
\hline & 6 & 0.9 & TE, AMC, IMP, CIP & \multirow{10}{*}{4} \\
\hline & 1 & 0.2 & IMP, CIP, CTX, CN & \\
\hline & 1 & 0.2 & TE, AMC, IMP, CN & \\
\hline & 1 & 0.2 & $\mathrm{TE}, \mathrm{AMC}, \mathrm{CTX}, \mathrm{CN}$ & \\
\hline & 1 & 0.2 & AMC, IMP, CTX, CN & \\
\hline & 1 & 0.2 & AMC, IMP, CIP, CN & \\
\hline & 32 & 4.9 & TE, AMC, IMP, CTX & \\
\hline & 4 & 0.6 & TE, AMC, CIP, CTX & \\
\hline & 9 & 1.4 & TE, IMP, CIP, CTX & \\
\hline & 7 & 1.1 & AMC, IMP, CIP, CTX & \\
\hline & 33 & 5.1 & TE, AMC, IMP, CIP, CTX & \multirow{5}{*}{5} \\
\hline & 1 & 0.2 & AMC, IMP, CIP, CTX, CN & \\
\hline & 4 & 0.6 & TE, AMC, IMP, CTX, CN & \\
\hline & 2 & 0.3 & TE, IMP, CIP, CTX, CN & \\
\hline & 1 & 0.2 & TE, AMC, CIP, CTX, CN & \\
\hline & 5 & 0.8 & TE, AMC, IMP, CIP, CTX, CN & 6 \\
\hline Total & 197 & 30.3 & & \\
\hline
\end{tabular}

$\mathrm{AMC}=$ amoxicillin-clavulanate $\mathrm{TE}=$ tetracycline $\mathrm{IMP}=$ imipenem $; \mathrm{CTX}=$ cefotaxime $; \mathrm{CIP}=$ ciprofloxacin $; \mathrm{CN}=$ gentamycin. 
Table 6. Resistance patterns of 70 MDR E. coli isolates from rodent samples.

\begin{tabular}{|c|c|c|c|c|}
\hline Rodent Samples & Number of Isolates (n) & Occurrence $(\%)$ & $\begin{array}{c}\text { Antibiotic Resistance } \\
\text { Patterns }\end{array}$ & $\begin{array}{c}\text { Number of } \\
\text { Antibiotic Classes }\end{array}$ \\
\hline \multirow[t]{18}{*}{$(\mathrm{n}=70)$} & 3 & 0.5 & AMC, IMP, CIP & \multirow{10}{*}{3} \\
\hline & 4 & 0.6 & AMC, CIP, CTX & \\
\hline & 1 & 0.2 & AMC, IMP, CTX & \\
\hline & 1 & 0.2 & IMP, CIP, CTX & \\
\hline & 8 & 1.2 & TE, IMP, CTX & \\
\hline & 1 & 0.2 & TE, AMC, IMP & \\
\hline & 1 & 0.2 & TE, CIP, CTX & \\
\hline & 1 & 0.2 & TE, AMC, CTX & \\
\hline & 1 & 0.2 & TE, AMC, IMP & \\
\hline & 3 & 0.5 & TE, IMP, CIP & \\
\hline & 2 & 0.3 & AMC, IMP, CIP, CTX & \multirow{5}{*}{4} \\
\hline & 9 & 1.4 & TE, AMC, IMP, CTX & \\
\hline & 1 & 0.2 & $\mathrm{TE}, \mathrm{AMC}, \mathrm{CIP}, \mathrm{CN}$ & \\
\hline & 3 & 0.5 & TE, IMP, CIP, CTX & \\
\hline & 5 & 0.8 & TE, AMC, IMP CIP & \\
\hline & 22 & 3.4 & TE, AMC, IMP, CIP, CTX & \multirow[b]{2}{*}{5} \\
\hline & 1 & 0.2 & TE, AMC, IMP, CIP, CN & \\
\hline & 3 & 0.5 & TE, AMC, IMP, CIP, CTX, CN & 6 \\
\hline Total & 70 & 10.8 & & \\
\hline
\end{tabular}

$\mathrm{AMC}=$ amoxicillin-clavulanate $\mathrm{TE}=$ tetracycline; $\mathrm{IMP}=$ imipenem $; \mathrm{CTX}=$ cefotaxime $; \mathrm{CIP}=$ ciprofloxacin $; \mathrm{CN}=$ gentamycin .

Table 7. Resistance patterns of 65 MDR E. coli isolates from soil samples.

\begin{tabular}{|c|c|c|c|c|}
\hline $\begin{array}{l}\text { Source of } \\
\text { Samples }\end{array}$ & Number of Isolates (n) & Occurrence $(\%)$ & $\begin{array}{c}\text { Antibiotic Resistance } \\
\text { Patterns }\end{array}$ & $\begin{array}{c}\text { Number of } \\
\text { Antibiotic Classes }\end{array}$ \\
\hline \multirow[t]{19}{*}{$(\mathrm{n}=512)$} & 1 & 0.2 & AMC, IMP, CIP & \\
\hline & 7 & 1.1 & AMC, IMP, CTX & \\
\hline & 3 & 0.5 & AMC, IMP, CTX & \\
\hline & 1 & 0.2 & IMP, CIP, CTX, CN & \\
\hline & 1 & 0.2 & IMP, CTX, CN & \\
\hline & 3 & 0.5 & TE, AMC, CTX & 3 \\
\hline & 1 & 0.2 & TE, AMC, CIP & \\
\hline & 1 & 0.2 & TE, CIP, CTX & \\
\hline & 2 & 0.3 & TE, IMP, CIP & \\
\hline & 13 & 2.0 & TE, IMP, CTX & \\
\hline & 12 & 1.8 & TE, AMC, IMP, CTX & \\
\hline & 4 & 0.6 & TE, IMP, CIP, CTX & \\
\hline & 4 & 0.6 & TE, AMC, CIP, CTX & 4 \\
\hline & 4 & 0.6 & AMC, IMP, CIP, CTX & \\
\hline & 1 & 0.2 & TE, IMP, CTX, CN & \\
\hline & 1 & 0.2 & AMC, IMP, CIP, CTX, CN & \\
\hline & 1 & 0.2 & TE, IMP, CIP, CTX, CN & 5 \\
\hline & 4 & 0.6 & TE, AMC, IMP, CIP, CN & \\
\hline & 1 & 0.2 & TE, AMC, IMP, CIP, CTX, CN & 6 \\
\hline Total & 65 & 10.0 & & \\
\hline
\end{tabular}

$\mathrm{AMC}=$ amoxicillin-clavulanate; $\mathrm{TE}=$ tetracycline; $\mathrm{IMP}=$ imipenem; $\mathrm{CTX}=$ cefotaxime; $\mathrm{CIP}=$ ciprofloxacin; $\mathrm{CN}=$ gentamycin .

\section{Discussion}

We conducted this study in Karatu because of high and frequent interactions among rodents, humans and chickens that have been reported in previous studies and shown to cause the spread of infections [16-20]. To the best of our knowledge, this is the first study in Tanzania to simultaneously screen MDR E. coli in chickens, humans, rodents 
and soil. In total, 650 out of 960 samples $(67.7 \%)$ were positive for $E$. coli. We found high resistance of isolates against imipenem $(79.8 \%)$, cefotaxime $(79.7 \%)$, tetracycline $(73.7 \%)$ and amoxicillin-clavulanate $(49.4 \%)$ compared to ciprofloxacin $(40.2 \%)$ and gentamicin $(9.7 \%)$. Indeed, the principal component 1 (PC1) indicated that isolates were more susceptible to gentamycin followed by ciprofloxacin, and relatively higher resistance rates of isolates to amoxicillin-clavulanate. The principal component 2 (PC2), indicated that tetracycline, imipenem, cefotaxime and amoxicillin-clavulanate were close to PC2 ( $Y$-axis) showing higher variances compared to other drugs, which implies higher resistance rates of isolates to these drugs. Interestingly, large positive loadings for tetracycline, imipenem and cefotaxime indicated greater and positive correlation between them in terms of resistance patterns. The overlapping of ellipses indicates that the proportions of resistant isolates did not vary significantly across sample sources, indicating widespread presence of AMR E.coli. Most of the highly resisted antibiotics are readily available and can be obtained over the counter without prescription [21-23].

We observed that $78.8 \%$ of all isolates were MDR E. coli, and that chicken $(27.7 \%)$ and human $(30.3 \%)$ isolates had significantly higher MDR isolates as compared to those recovered from rodents $(10.8 \%)$ and soil $(10.0 \%)$. Higher occurrence rates of MDR isolates in chickens and humans can be influenced by the frequent use and misuse of antibiotics in humans and poultry in Karatu [21,22,24,25]. The presence of MDR E. coli isolates in rodents indicates their potential role as hosts or vectors that can spread MDR E. coli to humans and chickens in Karatu and corresponds with other studies in Kenya, Germany, Canada and Vietnam [14,26-28] that associated rodents with carriage and spread of MDR and virulent $E$. coli strains in communities. The isolation frequency of MDR E. coli from soil was $10.0 \%$, which is close to the $12.6 \%$ reported in Bangladesh [29]. This indicates that household soils are potential reservoirs of MDR E. coli, possibly due to poor disposal of sewages and poultry manure in households. Previous studies in Tanzania have revealed that mismanagement of human and livestock wastes in households contaminates the soil with E. coli $[10,30,31]$. In our study, MDR isolates exhibited different resistance patterns. However, the combination of tetracycline, imipenem and cefotaxime (TE-IMP-CTX) was the most common in all types of samples, highlighting their frequent use in humans and poultry. The increased use of cefotaxime and tetracycline during disease treatment and prevention in poultry has been widely documented worldwide, resulting in widespread antibiotic resistance [32-35]. Interestingly, we found a higher isolation frequency of MDR E. coli in samples from Karatu $(21.1 \%)$, which is an urban area and district headquarter compared to Endabash ward (11.4\%), a rural area. Concomitant with this, we found a higher level of interaction between humans, chickens, rodents and their environment in urban households, coupled with a higher level of antimicrobial use and low levels of waste management, which may have facilitated the spread of MDR E. coli $[9,36]$. Finally, we acknowledge that our findings provide preliminary insight on the magnitude and pattern of AMR E. coli in humans, chickens, rodents and their environment and not transmission dynamics. Molecular studies of AMR genes of isolates from humans, chickens, rodents and soil will be required to determine cross-transmission of superbugs among different hosts and the environment in the area. Nonetheless, these findings should alert public health officials to take the necessary interventions, including raising public awareness, on the appropriate use of antimicrobial agents and proper hygiene measures, including waste disposal.

\section{Materials and Methods}

\subsection{Study Location}

The study was conducted in Karatu district in the Northern zone of Tanzania between June 2020 and March 2021. Karatu is located between latitudes $3^{\circ} 10^{\prime}$ and $4^{\circ} 00^{\prime} \mathrm{S}$ and longitude $34^{\circ} 47^{\prime}$ E. The district has a population of 230,166 people comprised of 117,769 men and 112,397 women with an average of 5 people per household [37]. Karatu has an altitude 
range of 1000 to $1900 \mathrm{~m}$ above sea level with two wet seasons annually (short rains between October and December and long rains from March to June).

\subsection{Sampling Strategy}

The study population included all households keeping local chickens, while the sampling frame was the list of these households. Five wards (Karatu, Endabash, Endamarariek, Mbulumbulu and Rhotia) were purposively selected based on population density (at least 16,000), number of households with chickens, and household size of at least 5 people. Households were randomly selected from a list provided by a livestock field officer at ward level by using a table of random numbers. At the household level, house owners' permission was used in trapping the rodents where areas for trapping in the surrounding environments relied on signs of rodents' activities. For each household, one adult (18 years and above) resident and one mature (7 months) scavenging chicken participated in microbiological sampling (1 fecal and 1 nasal swab). Additionally, at least one rodent (in-house rat, peridomestic rat or both) could be captured, as well as one soil sample collected per household. The selection of adult humans and mature chickens was based on the assumption that old individuals have been exposed to the interaction with rodents for a long time than young ones and hence are more likely to facilitate sharing of infections.

\subsection{Trapping of Rodents for Sample Collection}

Live trapping of 101 rodents was carried out using Modified-Sherman traps baited with peanut butter. Each captured rodent was euthanized by using di-ethyl-ether. Deep pharyngeal swabs and intestines were aseptically collected from the carcasses.

\subsection{Collection of Samples from Humans, Chickens and Soil}

A total of 960 samples was obtained from chickens (236), humans (243), rodents (101) and soil (290). Cloacal swabs were collected from randomly picked scavenging local chickens and human stools in households. Additionally, soil samples were randomly collected from five points in the household yards and mixed to compose 1 pooled soil sample [10]. Thereafter, all samples were stored in sterile containers at $-4^{\circ} \mathrm{C}$ and transported using Cary Blair transport medium to Tanzania Veterinary Laboratory Agency (TVLA) laboratory in Arusha for bacteriological analyses.

\subsection{Culture, Isolation and Identification of E. coli Isolates}

The specimens were plated onto MacConkey agar (Oxoid ltd., Detroid, MI, USA) and incubated aerobically at $37^{\circ} \mathrm{C}$ for $24 \mathrm{~h}$. Presumptive colonies of $E$. coli were subjected to a combination of four biochemical tests-indole, methyl red, Voges-Proskauer and citrate utilization (IMViC) — as well as motility tests for identification as per Quinn et al. [38]. E. coli strain American Type Culture Collection (ATCC) 29,522 was used as a standard organism.

\subsection{Antibiotic Susceptibility Testing of E. coli Isolates}

An antibiotic susceptibility test was performed by using Kirby-Bauer disc diffusion method on Mueller-Hinton Agar plates (Oxoid, Basingstoke, UK) with commercially available discs as described by [39]. The antibiotics tested were tetracycline (30 $\mu \mathrm{g})$, imipenem $(10 \mu \mathrm{g})$, chloramphenicol $(30 \mu \mathrm{g})$, gentamicin $(10 \mu \mathrm{g})$, ciprofloxacin $(5 \mu \mathrm{g})$, cefotaxime $(30 \mu \mathrm{g})$ and amoxicillin-clavulanate $(20 \mu \mathrm{g} / 10 \mu \mathrm{g})$. Pure colonies of the identified lactose fermenters were emulsified into $5 \mathrm{~mL}$ of sterile saline. The suspensions were adjusted to achieve turbidity equivalent to $0.5 \mathrm{McF}$ arland standard solutions, emulsified using sterile cotton swabs onto Mueller-Hinton Agar plate, and incubated at $37^{\circ} \mathrm{C}$ for 16 to $18 \mathrm{~h}$. After incubation the inhibition zone of each antimicrobial agent was measured, and results were interpreted according to the CLSI standards [39]. E. coli strain American Type Culture Collection (ATCC) 29,522 was used as standard organism. An isolate was considered to be multidrug-resistant if it was non-susceptible to three or more drugs from different classes of antibiotics [40]. 


\subsection{Statistical Analyses}

Isolation frequencies of $E$. coli and antibiotic resistance profiles of isolates were entered into Microsoft Excel version 2010 (Microsoft Corporation, Redmond, WA, USA) and their percentages calculated by descriptive statistics. Association between categorical variables was analyzed using Chi-square (Fisher's exact and Pearson's) test. Principal component analysis (PCA) was used to describe the distribution of $E$. coli resistant isolates with respect to their sample sources and antibiotic resistance profiles. PCA was performed using $\mathrm{R}$ Statistical Package Windows version 3.4.2. Statistical significance was accepted at $p<0.05$.

\section{Conclusions}

The level of antimicrobial resistance, including multi-drug-resistant $E$. coli seen in isolates from humans, chickens, rodents and soil raises the possibility of widespread transmission of resistance genes and bacteria in the studied area, with the possibility of causing infections that are difficult to treat. The antibiotics used in this study are the ones that are commonly used in the area for treating both human and animal infections, implying that they have limited success in their intended use. Comprehensive interventions, using a one-health approach, would be required to control the situation. Such measures should include improving (i) awareness of the community on judicious use of antimicrobial agents in humans and animals, (ii) house conditions and waste management and (iii) rodent control measures.

Author Contributions: Collection of samples, laboratory processing and data analysis (V.S.S.), supervision and verification of the analytical methods (M.I.N.M., A.S.K. and G.M.). Manuscript developed by V.S.S. with input from M.I.N.M., G.M. and A.S.K. All authors have read and agreed to the published version of the manuscript.

Funding: The study was funded by the Government of the United Republic of Tanzania and the World bank through the Africa Centre of Excellence for Innovative Rodent Pest Management and Biosensor Technology Development (IRPM and BTD) at Sokoine University of Agriculture (SUA).

Institutional Review Board Statement: The ethical clearance for the study was issued by the National Institute for Medical Research (NIMR) of Tanzania (NIMR/HQ/R.8a/Vol.IX/3386). NIMR is the national health research coordinating body that ensures all health research follows the national health ethics requirements. Sokoine University of Agriculture (SUA) Institutional Animal Care and Use Ccommittee (IACUC) approved the use of animals in this study. The permission to work in the study area was sought from the Regional Administrative Office, Arusha.

Informed Consent Statement: Informed verbal consent was obtained from all subjects involved in the study.

Data Availability Statement: The data presented in this study are available on request from the corresponding author. The data are not publicly available due to privacy restrictions.

Conflicts of Interest: The authors declare no conflict of interest.

\section{References}

1. Islam, M.; Nayeem, M.; Hasan, M.; Sobur, M.; Ievy, S.; Rahman, S.; Kafi, M.; Ashour, H.M.; Rahman, M. Virulence Determinants and Multidrug Resistance of Escherichia coli Isolated from Migratory Birds. Antibiotics 2021, 10, 190. [CrossRef]

2. Orubu, E.S.F.; Zaman, M.H.; Rahman, M.T.; Wirtz, V.J. Veterinary antimicrobial resistance containment in Bangladesh: Evaluating the national action plan and scoping the evidence on implementation. J. Glob. Antimicrob. Resist. 2020, 21, 105-115. [CrossRef] [PubMed]

3. Etienne, M.; Lefebvre, E.; Frebourg, N.; Hamel, H.; Pestel-Caron, M.; Caron, F. Antibiotic treatment of acute uncomplicated cystitis based on rapid urine test and local epidemiology: Lessons from a primary care series. BMC Infect. Dis. 2014, 14, 1-8. [CrossRef]

4. Nolan, L.K.; Vaillancourt, J.P.; Barbieri, N.L.; Logue, C.M. Colibacillosis. In Diseases of Poultry; John Wiley \& Sons, Inc.: Hoboken, NJ, USA, 2020; pp. 770-830. [CrossRef]

5. Subramanya, S.H.; Bairy, I.; Metok, Y.; Baral, B.P.; Gautam, D.; Nayak, N. Detection and characterization of ESBL-producing Enterobacteriaceae from the gut of subsistence farmers, their livestock, and the surrounding environment in rural Nepal. Sci. Rep. 2021, 11, 1-13. 
6. Ievy, S.; Islam, M.; Sobur, M.; Talukder, M.; Rahman, M.; Khan, M.F.R. Molecular detection of avian pathogenic Escherichia coli (APEC) for the first time in layer farms in Bangladesh and their antibiotic resistance patterns. Microorganisms 2020, 8, 1021. [CrossRef]

7. Desvars-Larrive, A.; Ruppitsch, W.; Lepuschitz, S.; Szostak, M.P.; Spergser, J.; Feßler, A.T.; Schwarz, S.; Monecke, S.; Ehricht, R.; Walzer, C. Urban brown rats (Rattus norvegicus) as possible source of multidrug-resistant Enterobacteriaceae and meticillinresistant Staphylococcus spp., Vienna, Austria, 2016 and 2017. Eurosurveillance 2019, 24, 1900149. [CrossRef]

8. Gwenzi, W.; Chaukura, N.; Muisa-Zikali, N.; Teta, C.; Musvuugwa, T.; Rzymski, P.; Abia, A.L.K. Insects, Rodents, and Pets as Reservoirs, Vectors, and Sentinels of Antimicrobial Resistance. Antibiotics 2021, 10, 68. [CrossRef]

9. Hassell, J.M.; Ward, M.J.; Muloi, D.; Bettridge, J.M.; Robinson, T.P.; Kariuki, S.; Ogendo, A.; Kiiru, J.; Imboma, T.; Kang'ethe, E.K.; et al. Clinically relevant antimicrobial resistance at the wildlife-livestock-human interface in Nairobi: An epidemiological study. Lancet Planet. Health 2019, 3, e259-e269. [CrossRef]

10. Lupindu, A.M.; Dalsgaard, A.; Msoffe, P.L.; Ngowi, H.A.; Mtambo, M.M.; Olsen, J.E. Transmission of antibiotic-resistant Escherichia coli between cattle, humans and the environment in peri-urban livestock keeping communities in Morogoro, Tanzania. Prev. Vet. Med. 2015, 118, 477-482. [CrossRef] [PubMed]

11. Zhang, Y.-J.; Hu, H.-W.; Gou, M.; Wang, J.-T.; Chen, D.; He, J.-Z. Temporal succession of soil antibiotic resistance genes following application of swine, cattle and poultry manures spiked with or without antibiotics. Environ. Pollut. 2017, 231, 1621-1632. [CrossRef] [PubMed]

12. Sanderson, H.; Brown, R.S.; Hania, P.; McAllister, T.A.; Majury, A.; Liss, S.N. Antimicrobial resistant genes and organisms as environmental contaminants of emerging concern: Addressing global public health risks. In Management of Emerging Public Health Issues and Risks; Elsevier: Amsterdam, The Netherlands, 2019; pp. 147-187.

13. Touati, M.; Hadjadj, L.; Berrazeg, M.; Baron, S.A.; Rolain, J.M. Emergence of Escherichia coli harbouring $m c r-1$ and $m c r-3$ genes in North West Algerian farmlands. J. Glob. Antimicrob. Resist. 2020, 21, 132-137. [CrossRef] [PubMed]

14. Guenther, S.; Bethe, A.; Fruth, A.; Semmler, T.; Ulrich, R.G.; Wieler, L.H.; Ewers, C. Frequent combination of antimicrobial multiresistance and extraintestinal pathogenicity in Escherichia coli isolates from urban rats (Rattus norvegicus) in Berlin, Germany. PLoS ONE 2012, 7, e50331. [CrossRef]

15. Feng, A.Y.; Himsworth, C.G. The secret life of the city rat: A review of the ecology of urban Norway and black rats (Rattus norvegicus and Rattus rattus). Urban Ecosyst. 2014, 17, 149-162. [CrossRef]

16. Kilonzo, B.; Mbise, T.; Mwalimu, D.; Kindamba, L. Observations on the endemicity of plague in Karatu and Ngorongoro, northern Tanzania. Tanzan. J. Health Res. 2006, 8, 1-6. [CrossRef]

17. Makundi, R.H.; Massawe, A.W.; Borremans, B.; Laudisoit, A.; Katakweba, A. We are connected: Flea-host association networks in the plague outbreak focus in the Rift Valley, northern Tanzania. Wildl. Res. 2015, 42, 196-206. [CrossRef]

18. Ziwa, M.H.; Matee, M.I.; Hang'ombe, B.M.; Lyamuya, E.F.; Kilonzo, B.S. Plague in Tanzania: An overview. Tanzan. J. Health Res. 2013, 15. [CrossRef]

19. Haule, M.; Lyamuya, E.E.; Hang'ombe, B.M.; Matee, M.I. Investigation of Fleas as Vectors in the Transmission of Plague during a Quiescent Period in North-Eastern, Tanzania; Sokoine University of Agriculture: Morogoro, Tanzania, 2013.

20. Makundi, R.H.; Massawe, A.W.; Mulungu, L.S.; Katakweba, A.; Mbise, T.J.; Mgode, G. Potential mammalian reservoirs in a bubonic plague outbreak focus in Mbulu District, northern Tanzania, in 2007. Mammalia 2008, 72, 253-257. [CrossRef]

21. Rugumisa, B.; Call, D.R.; Mwanyika, G.O.; Subbiah, M.; Buza, J. Comparison of the Prevalence of Antibiotic-Resistant Escherichia coli Isolates from Commercial-Layer and Free-Range Chickens in Arusha District, Tanzania. 2016. Available online: https: / / dspace.nm-aist.ac.tz/handle/20.500.12479/457 (accessed on 20 June 2021).

22. Lyimo, B.; Buza, J.; Subbiah, M.; Smith, W.; Call, D.R. Comparison of antibiotic resistant Escherichia coli obtained from drinking water sources in northern Tanzania: A cross-sectional study. BMC Microbiol. 2016, 16, 254. [CrossRef]

23. Caudell, M.A.; Mair, C.; Subbiah, M.; Matthews, L.; Quinlan, R.J.; Quinlan, M.B.; Zadoks, R.; Keyyu, J.; Call, D.R. Identification of risk factors associated with carriage of resistant Escherichia coli in three culturally diverse ethnic groups in Tanzania: A biological and socioeconomic analysis. Lancet Planet. Health 2018, 2, e489-e497. [CrossRef]

24. Kimera, Z.I.; Mgaya, F.X.; Misinzo, G.; Mshana, S.E.; Moremi, N.; Matee, M.I. Multidrug-Resistant, Including Extended-Spectrum Beta Lactamase-Producing and Quinolone-Resistant, Escherichia coli Isolated from Poultry and Domestic Pigs in Dar es Salaam, Tanzania. Antibiotics 2021, 10, 406. [CrossRef] [PubMed]

25. Mgaya, F.X.; Matee, M.I.; Muhairwa, A.P.; Hoza, A.S. Occurrence of multidrug resistant Escherichia coli in raw meat and cloaca swabs in poultry processed in slaughter slabs in Dar es Salaam, Tanzania. Antibiotics 2021, 10, 343. [CrossRef]

26. Gakuya, F.; Kyule, M.; Gathura, P.; Kariuki, S. Antimicrobial susceptibility and plasmids from Escherichia coli isolated from rats. East Afr. Med. J. 2001, 78, 518-522. [CrossRef]

27. Himsworth, C.G.; Zabek, E.; Desruisseau, A.; Parmley, E.J.; Reid-Smith, R.; Jardine, C.M.; Tang, P.; Patrick, D.M. Prevalence and characteristics of Escherichia coli and Salmonella spp. in the feces of wild urban Norway and black rats (Rattus norvegicus and Rattus rattus) from an inner-city neighborhood of Vancouver, Canada. J. Wildl. Dis. 2015, 51, 589-600. [CrossRef]

28. Le Huy, H.; Koizumi, N.; Ung, T.T.H.; Le, T.T.; Nguyen, H.L.K.; Hoang, P.V.M.; Nguyen, C.N.; Khong, T.M.; Hasebe, F.; Haga, T. Antibiotic-resistant Escherichia coli isolated from urban rodents in Hanoi, Vietnam. J. Vet. Med. Sci. 2020, 82, 653-660. [CrossRef] 
29. Montealegre, M.C.; Roy, S.; Böni, F.; Hossain, M.I.; Navab-Daneshmand, T.; Caduff, L.; Faruque, A.; Islam, M.A.; Julian, T.R. Risk factors for detection, survival, and growth of antibiotic-resistant and pathogenic Escherichia coli in household soils in rural Bangladesh. Appl. Environ. Microbiol. 2018, 84, e01978-18. [CrossRef] [PubMed]

30. Badowski, N.; Castro, C.M.; Montgomery, M.; Pickering, A.J.; Mamuya, S.; Davis, J. Understanding household behavioral risk factors for diarrheal disease in Dar es Salaam: A photovoice community assessment. J. Environ. Public Health 2011, $2011,130467$. [CrossRef]

31. Pickering, A.J.; Julian, T.R.; Marks, S.J.; Mattioli, M.C.; Boehm, A.B.; Schwab, K.J.; Davis, J. Fecal contamination and diarrheal pathogens on surfaces and in soils among Tanzanian households with and without improved sanitation. Environ. Sci. Technol. 2012, 46, 5736-5743. [CrossRef] [PubMed]

32. Sadiq, M.; Syed-Hussain, S.; Ramanoon, S.; Saharee, A.; Ahmad, N.; Zin, N.M.; Khalid, S.; Naseeha, D.; Syahirah, A.; Mansor, R. Knowledge, attitude and perception regarding antimicrobial resistance and usage among ruminant farmers in Selangor, Malaysia. Prev. Vet. Med. 2018, 156, 76-83. [CrossRef] [PubMed]

33. Teklu, D.S.; Negeri, A.A.; Legese, M.H.; Bedada, T.L.; Woldemariam, H.K.; Tullu, K.D. Extended-spectrum beta-lactamase production and multi-drug resistance among Enterobacteriaceae isolated in Addis Ababa, Ethiopia. Antimicrob. Resist. Infect. Control 2019, 8, 39. [CrossRef]

34. Mdegela, R.H.; Mwakapeje, E.R.; Rubegwa, B.; Gebeyehu, D.T.; Niyigena, S.; Msambichaka, V.; Nonga, H.E.; Antoine-Moussiaux, N.; Fasina, F.O. Antimicrobial Use, Residues, Resistance and Governance in the food and agriculture sectors, Tanzania. Antibiotics 2021, 10, 454. [CrossRef]

35. Sindato, C.; Mboera, L.E.; Katale, B.Z.; Frumence, G.; Kimera, S.; Clark, T.G.; Legido-Quigley, H.; Mshana, S.E.; Rweyemamu, M.M.; Matee, M. Knowledge, attitudes and practices regarding antimicrobial use and resistance among communities of Ilala, Kilosa and Kibaha districts of Tanzania. Antimicrob. Resist. Infect. Control 2020, 9, 194. [CrossRef] [PubMed]

36. Arnold, K.E.; Williams, N.J.; Bennett, M. 'Disperse abroad in the land': The role of wildlife in the dissemination of antimicrobial resistance. Biol. Lett. 2016, 12, 20160137. [CrossRef] [PubMed]

37. URT (United Republic of Tanzania). 2012 Population and Housing Census_Population Distribution by Administrative Areas; URT: Dodoma, Tanzania, 2013; p. 244.

38. Quinn, P.J.; Markey, B.K.; Carter, M.E.; Donnelly, W.J.; Leonard, F.C. Veterinary Microbiology and Microbial Disease: Pathogenic Bacteria Blackwell Scientific Publications; Oxford: London, UK, 2002; pp. 113-115.

39. CLSI. Clinical and Laboratory Standards Institute-Performance Standards for Antimicrobial Susceptibility Testing; CLSI: Wayne, PA, USA, 2020.

40. Magiorakos, A.-P.; Srinivasan, A.; Carey, R.; Carmeli, Y.; Falagas, M.; Giske, C.; Harbarth, S.; Hindler, J.; Kahlmeter, G.; OlssonLiljequist, B. Multidrug-resistant, extensively drug-resistant and pandrug-resistant bacteria: An international expert proposal for interim standard definitions for acquired resistance. Clin. Microbiol. Infect. 2012, 18, 268-281. [CrossRef] [PubMed] 